\title{
A Qualitative Study of Depression in Primary Care: Missed Opportunities for Diagnosis and Education
}

\author{
Barry G. Saver, MD, MPH, Victoria Van-Nguyen, BA, Gina Keppel, BA, and \\ Mark P. Doescher, MD, MSPH
}

Purpose: Depression is one of the most commonly encountered chronic conditions in primary care, yet it remains substantially underdiagnosed and undertreated. We sought to gain a better understanding of barriers to diagnosis of and entering treatment for depression in primary care.

Methods: We conducted and analyzed interviews with 15 subjects currently being treated for depression recruited from primary care clinics in an academic medical center and an academic public hospital. We asked about experiences with being diagnosed with depression and starting treatment, focusing on barriers to diagnosis, subject understanding of depression, and information issues related to treatment decisions.

Results: Subjects reported many visits to primary care practitioners without the question of depression being raised. The majority had recurrent depression. Many reported that they did not receive enough information about depression and its treatment options. In the majority of cases, practitioners decided the course of treatment with little input from the patients.

Conclusions: In this sample of depressed patients, we found evidence of frequent missed diagnoses, substantial information gaps, and limited patient understanding and choice of treatment options. Quality improvement efforts should address not only screening and follow-up but patient education about depression and treatment options along with elicitation of treatment preferences. ( $\mathrm{J}$ Am Board Fam Med 2007;20:28-35.)

Depression is a common mental health problem leading to significant morbidity and mortality and high medical and societal costs. ${ }^{1-3}$ The World Health Organization estimated that major depression caused more disability worldwide in 1990 than ischemic heart disease or cerebrovascular disease. ${ }^{4}$ The prevalence of major depressive disorders in the US population aged 18 years and older has been estimated at $5 \%,{ }^{5}$ and it is one of the most commonly encountered conditions in primary care, ${ }^{6,7}$ but up to $50 \%$ of cases go unrecognized. ${ }^{8,9}$ In many

This article was externally peer-reviewed.

Submitted 8 February 2006; revised 27 September 2006; accepted 5 October 2006.

From the Department of Family Medicine (BGS, VV-N, GK, MPD), School of Education (VV-N), and School of Public Health (GK), University of Washington, Seattle, WA.

Prior presentation: A portion of this work was presented at the 33rd North American Primary Care Research Group Annual Meeting, Quebec City, Quebec, October 15-18, 2005.

Conflict of interest: none reported.

Corresponding author: Barry G. Saver, MD, MPH, Department of Family Medicine and Community Health, University of Massachusetts Medical School, 55 Lake Avenue North, Benedict Building A3-146, Worcester, MA 01655 (E-mail: Barry.Saver@umassmed.edu). cases, it is more appropriately viewed as a chronic disorder with remissions and relapses than an acute illness. $^{10-12}$

Even if diagnosed, care for depression is frequently flawed. Many persons diagnosed with depression do not commence treatment for it, ${ }^{9}$ and the majority of persons who do enter treatment do not receive their preferred type of treatment, even though this seems to lead to better outcomes. ${ }^{13,14}$ Furthermore, many persons starting treatment do not complete an adequate treatment course. ${ }^{15}$ The US Preventive Services Task Force "recommends screening adults for depression in clinical practices that have systems in place to assure accurate diagnosis, effective treatment, and follow-up" with a grade B recommendation, ${ }^{16}$ as such systems have been demonstrated to improve health status and, in some instances, to reduce health care costs. ${ }^{17-20}$

There is a remarkable paucity of information about patients' understanding of depression and its treatment options, and the role patients play in choosing treatment options. The very limited evidence available suggests quite modest benefits of patient education materials for depression in isolation from more comprehensive interventions. ${ }^{21,22}$ 
Some studies have included patient informational materials as part of a systematic intervention, but not evaluated them separately. ${ }^{14,17,19}$ In some cases, patient education materials may have more of a medical than a patient-centered orientation and may not address a number of patients' key questions. ${ }^{23}$

In this study, we report results of qualitative analyses of interviews with patients currently under treatment for depression about their experiences with being diagnosed with and starting treatment for depression. We specifically sought to explore their understanding of depression before and after receiving the diagnosis, sources and adequacy of information about depression and its treatment options, and their roles in choosing treatment options. We chose a qualitative approach because of the limited extant information in this area to "map the terrain" and be open to unexpected findings.

\section{Methods}

\section{Participant Recruitment}

Subjects were recruited by posting flyers in primary care clinics affiliated with the University of Washington Medical Center, an academic medical center, and at Harborview Medical Center, a public/ teaching hospital affiliated with the University of Washington. We advertised for persons who had been diagnosed with depression within the past year. Persons who had been diagnosed with depression, spoke English, and were able to give informed consent were eligible to participate in the study. This study was approved by the University of Washington's Human Subjects Institutional Review Board.

\section{Conduct of Interviews}

We conducted semistructured, qualitative interviews lasting 30 to 60 minutes with 15 subjects currently undergoing treatment for depression. Subjects were interviewed by 2 study members, with one acting as primary interviewer and the other focusing more on detailed note taking, with the exception of 3 cases where only 1 team member was available. A family physician-investigator (BGS) participated in 11 of the 15 interviews. The backgrounds of the other interviewers were education $(\mathrm{VV}-\mathrm{N})$ and public health (GK). Each interviewer took typewritten notes during the interviews, touch typing and maintaining eye contact with subjects and only rarely asking for brief pauses to catch up on note taking. Notes were checked for consistency and merged after the interviews.

Interviews were structured around a set of root questions covering subjects' experience of and perspectives on being diagnosed with depression, receiving information about the diagnosis, receiving information about treatment options, and deciding to start treatment for depression. Each root question was followed by a number of probe questions to flesh out detail in subjects' responses. Subjects were asked about their current episode of depression and any previous episodes. The template for the root and probe questions is contained in the Appendix.

\section{Data Analysis}

We used a qualitative descriptive approach ${ }^{24}$ based on our interview template. Using notes from the first 3 interviews, 3 researchers (BGS, VV-N, and GK) who participated in the interviewing process independently classified statements according to this schema, first into the major categories underlying our interview template and then into subcategories as we identified them in the data. We added new categories and subcategories for statements not fitting this framework, and searched for themes and concepts common to these interviews. These 3 researchers then compared results and, through an interactive process of discussion, reflection, and scrutiny of the interview template, interview notes, and initial coding, arrived at consensus on a refined coding scheme. Using this coding scheme, 2 researcher-interviewers ( VV-N and GK) independently coded the remaining interview notes using QSR NVivo 2.0 (Qualitative Systems and Research, Australia, 2002) and met to compare different perspectives and interpretations of ambiguous data and identification of concepts not covered by the coding scheme; differences were reconciled with no significant disagreements. The other researcher-interviewer (BGS) reviewed all the notes and coding for consistency and to ensure that no significant concepts or themes had been overlooked or statements misclassified or left uncoded. This resulted in coding of a few additional statements with the existing coding scheme but no new categories or disagreements with the coding assignments.

Given our a priori descriptive goals, we did not seek to generate new theories about depression in primary care; rather, we focused on describing common experiences and identifying gaps and bar- 
riers that might be amenable to interventions to improve depression care for future patients.

\section{Results}

The 15 participants ranged in age from their mid20 s to their late 50 s, with about half in their mid40 s, and were evenly split between males and females. There were 7 Caucasian, 6 African American, 1 Asian American, and 1 Native American subjects. Six participants reported college or graduate degrees, and 6 more reported some college education. Four subjects mentioned a diagnosis of bipolar disorder, 3 reported anxiety disorders, and 5 gave histories of substance use problems. We chose to retain and analyze interviews with participants reporting a diagnosis of bipolar disorder, because their reported experiences with depression diagnosis and treatment were quite similar to those of our participants not reporting a bipolar diagnosis, and depression is a common presentation of bipolar disorder. Similarly, we have retained the participants in our sample who reported histories of substance use as the issues raised by these participants were very much the same as those raised by other participants, with the addition of active substance use being a barrier to willingness to undertake treatment for depression.

A majority of our participants reported being diagnosed with depression by a mental health care practitioner, but a substantial minority reported receiving an initial diagnosis from a primary care practitioner (PCP). One third reported their initial diagnosis came as a result of an emergency department visit. All subjects reported limited understanding of depression before their initial diagnosis. None reported having, before diagnosis, acquaintance with views of depression as a frequently heritable condition involving neurotransmitters in the brain, regardless of their educational attainment. Sample comments illustrating subjects' reported understanding of depression before their initial diagnoses are presented in Table 1.

Participants frequently reported incidents of missed diagnosis during visits to PCPs. As exemplified by quotes in Table 2, reasons cited for missed diagnoses fell into 2 categories: patientrelated and practitioner-related factors. Some subjects expressed either inability or unwillingness to raise the issue with their practitioners. Others reported that practitioners were unsuspecting, focus-
Table 1. Participant Statements about Prediagnosis Understanding of Depression

- I don't think I had much of an understanding. I knew for myself though how it felt-it wasn't good.

- I didn't really have a concept of it. I had heard of people who had trouble getting out of bed but I didn't graspknow what it meant.

- Initially, I was embarrassed. I thought it was laziness, ignorance, something I could change. That lasted for a pretty long time.

- Suicide. That's it. Basically, I have seen people depressed, and I didn't understand it until it happened to me. I thought it was so bad that I would end up killing myself.

- My understanding of depression was that one got depressed because of a culmination of events in their life that they normally just blew off or denied ever happened. The shadow of those events would move in.

ing on the subjects' somatic complaints (depression-related or otherwise), seemed uninterested in the possibility of nonphysical issues, or were frankly dismissive of the diagnosis when the subject raised it with them.

Only a few participants reported receiving helpful verbal information from their PCPs, also in some cases accompanied by being referred to a patient information library; most reported receiving little or no information from their PCPs about depression. Of the 13 participants who had seen a

\section{Table 2. Participant Statements about Missed Diagnoses of Depression}

Patient unwilling or unable to raise the question of depression:

- I never knew how to express it, and I had a shame in expressing it. I felt the shame in mentioning it to my family, even though my family had a whole depression issue. So if I couldn't talk to my family, then how could I have talked about it to a doctor?

- I was good at hiding it, until I just fell apart one day.

Provider unsuspecting, disinterested, or dismissive:

- I had no indication that they were thinking about that while they were trying out other things. I presented myself with physical symptoms, and that was it.

- I think that because the subject was about my pain, other matters at hand, that they didn't ask about my sleeping habits, my mood, appetite. My doctor couldn't have known.

- I guess maybe I wasn't really sure what was going on. And so, I probably didn't mention it as problems I was having, and I perceived (my primary care doctor) as someone more interested in physical issues.

- I had one person tell me it was all in my head, that there was nothing anyone could help.

- When I mentioned it to my primary care doctor, he said, "Well, you smile-you don't seem to be depressed." So I just stopped mentioning it; that was about 2 years ago. 
mental health care practitioner at any point, a majority reported receiving information about depression; a minority felt the information they received from mental health professionals was inadequate. Some subjects reported seeking out information from other sources, such as books, broadcast media, and the internet. Representative comments about sources of information about the diagnosis of depression are shown in Table 3.

A number of subjects reported having received written information, but this information was frequently recalled as not very helpful. The participants who reported doing their own research about depression proved more informed about current understanding of the genetics and neurobiology of depression than those who had not. Two persons specifically commented that learning about this helped them stop blaming themselves for their depression (see quotes in Table 3).

Many participants reported barriers to obtaining information about depression and its treatment. These could be grouped into several categories: lack of motivation due to depression, stigma of depression and/or denial of the diagnosis, practitioners seeming unresponsive, and a mismatch between their preferred mode of learning and how information was offered. Quotes exemplifying these issues are provided in Table 4.

Fewer than half of our subjects reported being given any information about counseling as a treatment option. None remembered being given any explanation of what counseling options, such as cognitive-behavioral therapy, problem-solving therapy, and interpersonal therapy, might be like.

Only a minority of the participants indicated that they felt they had had some say in their treatment decisions and even where they did, not all felt they had sufficient information to make a good choice. However, not all wanted to make this choice, with some preferring to trust their practitioners to make the choice. Quotes pertaining to issues of role in choice of therapy are shown in Table 5.

\section{Discussion}

Because almost $75 \%$ of patients who seek help for depression do so in primary care settings, ${ }^{3}$ substantial improvement in the quality of care for depression must address deficits in primary care. Our interviews highlight some key areas where depres-
Table 3. Participant Statements Regarding Sources of Information about Depression

Information from primary care providers:

- None. Not in the past either.

- Quite frankly, [PCP told me] nothing. I just told them what I felt, and they identified it as depression. They didn't go into the details, and I didn't ask for the information.

- I actually don't remember too much. She said that it is nothing to get upset about, and that it is common and she will get help for it.

- She $[\mathrm{PCP}]$ referred me to one of the patient libraries. So I went there and got some info on depression and taking medications and how that should help and what to do if it doesn't.

- I had one person tell me it was all in my head, there was nothing anyone could help. That's why I went to [mental health clinic]. I knew they had counseling and medication.

Information from mental health providers:

- I was given some brochures, but they seem like they're written for sixth graders.

- I was seeing a psychiatrist; they explained about my depression and why I got it.

- I was given some literature to read and I had discussions with my counselor about it . . No more information except research on my own.

- They [mental health services agency] gave me some flyers that I read. They asked me which category fit me best.

- The second doctor was very good at gauging my personality, figuring out the best way to talk to me.

- I got a lot of information from the psychiatrist I saw.

Other sources of information:

- I remember going to bookstores and reading.

- My ears would pick up on things if I heard a show on $T V$, radio, a new article in a magazine

- I went on the Internet, Web MD, looking for what the symptoms were, what I should be looking for, if it was triggered from a traumatic experience.

- Just recently heard about synapses, serotonin, what they do. I guess maybe on the Internet I learned it was hereditary, so I can blame my mom and dad. . . . I think the more I learn about it, the more I understand it and can deal with it instead of just shutting down and assuming I'll always be depressed. I like learning the scientific reasons for it.

- I've seen that ad on TV-the little bouncing head, talking about things not getting from one synapse to another; I do understand there's an organic element there. That's nice to know, otherwise I could just blame myself. I learned more from watching that commercial on television [than from my provider].

- As far as information about medicine and whatnot, I'd heard of Prozac, Paxil; I really do my research by asking people I know. . . . Media is a very powerful tool. All those commercials I used to make fun of, I understand now-the [drug name] commercials, ...

sion care might be improved: screening for depression; patient education about current understanding of depression and treatment options; improving provider attitudes and knowledge about depression 
Table 4. Participant Statements about Barriers to Getting Information about Depression

Lack of motivation to learn when depressed:

- It [getting information from provider] would have been very helpful. I could have looked by myself or gone to the library. But when you are depressed, you just want things handed to you.

- They didn't go into the details, and I didn't ask for the information. I don't want to blame them for not telling me more, because I didn't invite or encourage them to share.

- I was seeing a psychiatrist, they explained about my depression and why I got it. I wasn't focusing on that much. I was thinking about a better way to kill myself.

Stigma, denial:

- I was afraid of counseling because I felt that my family would disown me. I ended up taking medication and I was afraid of dealing with people about this.

- I guess shame, embarrassment. The Internet is better-I can just do it on my own.

- Not wanting to just accept that that was going on. You have to get a job, move on, maintain some kind of normality.

- She said, "When you're ready, I've got a whole bunch of stuff you might want to read, you might find yourself in some of it." She'd start slowly with the uncomfortable stuff. I just wasn't ready to hear it.

Limited provider responsiveness:

- I would have liked to know how medication or counseling, both would work for me. But the doc was like she was doctor and she would know better.... They are not able to show full concern because they are low in staff and funding. The frustration shows because you have to make it clear that you need more than this. For a patient who did not know how to make it clear, you are in a Catch-22.

- The first counselor referred me to some pamphlets, kind of brushed over the whole subject. He seemed like he had reached a level of detachment with his job. Just totally unmemorable.

- I did try to talk to doctors about depression. It just wasn't working. I let my doctors know that. That doctor kept trying to tell me this was the best thing for me; I know there are other medications.

Information-seeking style:

- I would never sit down and read something about medicine. It has never interested me. I learned more from watching that commercial on television.

- I don't seek out information, but if I come across it, then I will take it and read it.

and its treatment where there are gaps; and increasing the collaborative nature of decision making about treatment options.

Our participants reported frequent missed diagnoses, even among persons with recurrent depression. Although one might debate the value of universal screening, particularly in the widespread absence of "systems in place to assure accurate diagnosis, effective treatment, and follow-up,"16 use of practice-based information systems to imple-
Table 5. Participant Statements about Participation in Treatment Decisions

No perceived choice:

- They just handed me a drug and said go on it right now... I felt rushed along, given a prescription, told this will fix it.

- We never discussed any treatment plans. They just diagnosed and then gave me Zoloft and that's it.

Lack of sufficient information to make a choice:

- I just got the option that we got medication or counseling available. I don't know what's required of you and it was scary. I didn't know how to ask any of these questions.

- Just about medication. I don't really feel like there were options except for the different types of medications, which might have different side effects. And I might decide which side effects I potentially have to deal with. It did seem that there were not any psychological components brought up.

Trust practitioner to make the choice:

- I trust my doctor implicitly, so I didn't question. I felt perhaps that she was making the best choice based on her knowledge of me, which was pretty vast. ... I would probably like to see what other treatment options are. I fear getting confused by the onslaught of treatment options.

- I've always been like going to a car mechanic-'Treat me. Just do what you need to do.'

Made an informed choice:

- Yes, when we had the initial interview, he [PCP] left that open and up to me, whether to have some concurrent counseling along with medication or just try the medication. He left that up to me and facilitated that role or would refer me to someone else, whatever I wanted.

ment screening for recurrent depression among persons with past diagnoses might well be efficient and cost-effective in the current practice environment.

As noted above, the limited evidence on educational interventions for depression ${ }^{21,22}$ does not suggest that education alone is likely to be particularly effective in improving outcomes of depression treatment. However, education is a key component of self-management support, facilitating patients in taking active roles in commencing and continuing treatment. Our participants reported that they generally received limited information from practitioners about depression and the treatment options available, both in primary care and mental health care settings. Most reported that they would have liked more information. A number of barriers to obtaining information were reported, with both patient-related and practitioner-related factors appearing important.

Although this study did not specifically address stigma associated with depression, several of our 
participants raised this issue. and other studies have shown that stigma is a major barrier to diagnosis and treatment of depression. ${ }^{3,25-28}$ Education may help to reduce personal stigma associated with depression. $^{22}$

Most of our participants reported that they would have liked more say in their choice of treatments. Implementing systems to solicit treatment preferences of persons diagnosed with depression and coordination with counseling resources could substantially improve outcomes given the evidence that providing patients with their preferred mode of treatment increases both treatment uptake and adherence $^{13,14}$ and that more patients seem to desire counseling versus medication for treatment of depression. ${ }^{29-31}$

A number of limitations of this study must be acknowledged. First, being based on a limited number of interviews with volunteer participants recruited from outpatient clinics at 2 hospitals associated with a single teaching institution, we cannot know how well our finding generalize to the universe of persons under treatment for depression in the US. Both sites probably have an overrepresentation of persons with significant, chronic mental health issues. Volunteers may well differ in many ways from "average" patients with depression. Second, recall of our participants may have been incomplete and/or biased. Depression can interfere with both memory and motivation, so it is possible that some subjects received information and/or choices that they did not recall during the interviews. Third, as with most research, our findings and conclusions are subject to potential bias from our preconceptions and prejudices. Our budget did not permit transcription of interview recordings and we did not record the interviews, so choices about what to record in our notes could influence our findings. However, having 2 persons simultaneously take notes in 12 of the 15 cases should have minimized effects of selective recording of information. Fourth, having only interviewed persons engaged in active treatment for depression, we cannot generalize our findings to those who declined or stopped therapy. Fifth, we had anticipated our participants would primarily be persons who had received new diagnoses of depression in the primary care setting within the previous year, but many had chronic or recurrent depression and were seeing or had seen mental health practitioners. Our interview template did not have probes to clearly delineate the separate roles of primary care and mental health specialty care and the coordination of care between them. Sixth, we have no way of knowing whether addressing the shortcomings in diagnosis and patient education about depression we have identified will, in fact, improve outcomes of depression treatment.

However, our findings are consistent with existing literature on shortcomings in diagnosis and treatment of depression. Addressing these deficits would enhance the patient-centeredness of care for depression and offers the potential to improve engagement with and outcomes of treatment for depression.

\section{Appendix}

I. Background Information

1. Age

2. Gender

3. Ethnicity

4. Education

5. Years diagnosed with depression

II. Diagnosis

6. How was your depression diagnosed?

a. Did you go to the doctor specifically because you thought you were depressed and wanted help?

b. If not, what made you go to the doctor?

c. Had you been to the doctor earlier, while you were depressed, without having the question of depression brought up?

d. If so, why do you think it didn't come up?

e. Had you been diagnosed with or treated for depression previously?

III. Recognizing Depression:

7. What was your understanding of depression before you were diagnosed?

IV. Source of information/understanding depression

8. What information were you given about depression?

a. What information were you given when the diagnosis was made?

b. How was it given? For example, did the doctor talk with you to help you understand about depression? Were you given written materials? Re- 
ferred to books, web resources, or something else?

c. Did you feel you had enough information?

d. If not, what else did you want to know?

e. What prevented you from learning more? (For example, no time left in the visit, information was too hard to understand at the time, information was just not offered, or other.)

$\mathrm{V}$. Information on treatment options

9. Did you feel you were given enough information about treatment options to make a good choice?

a. Did you learn about both counseling and medications?

b. Did you understand what counseling for depression generally involves?

c. Did you hear about the different types of antidepressant medications (such as tricyclic antidepressants and SSRIs) and the pros and cons of each?

d. How was the information provided? For example, did the doctor talk with you? Were you given written materials? Referred to books, web resources, or something else?

e. Did you feel you had enough information to make a good choice?

f. If not, what else would you have liked to know?

VI. Treatment decision

10. After the discussion of depression as a diagnosis, did you decide to start treatment?

a. If not then why not?

b. Did you later start treatment? If so, why?

c. What had changed?

d. If no change then explain why?

VII. Continued treatment

11. If you started treatment, how soon after this was your next contact with your provider?

12. Did you initiate the contact or did they? (E.g., someone from your doctor's office called you or they scheduled a follow-up appointment a week or two after you decided to start treatment.)
VIII. Closing

13. Do you have any thoughts about things that would have improved the diagnosis and treatment of your depression? This might be helping it be diagnosed sooner, helping you understand the diagnosis better, helping you understand treatment options better, helping you start treatment, or helping you stick with treatment.

14. Do you have any other thoughts about how doctors can do a better job helping people with depression?

\section{References}

1. Simon G, Ormel J, VonKorff M, Barlow W. Health care costs associated with depressive and anxiety disorders in primary care. Am J Psychiatry 1995;152:352-7.

2. Stewart WF, Ricci JA, Chee E, Hahn SR, Morganstein D. Cost of lost productive work time among US workers with depression. JAMA 2003;289:313544.

3. Goldman LS, Nielsen NH, Champion HC. Awareness, diagnosis, and treatment of depression. J Gen Intern Med 1999;14:569-80.

4. Murray CJ, Lopez AD. Alternative projections of mortality and disability by cause 1990-2020: Global Burden of Disease Study. Lancet 1997;349:1498504.

5. Regier DA, Narrow WE, Rae DS, et al. The de facto US mental and addictive disorders service system: epidemiologic catchment area prospective 1-year prevalence rates of disorders and services. Arch Gen Psychiatry 1993;50:85-94.

6. Nelson C, Woodwell D. National Ambulatory Medical Care Survey: 1993 summary. Vital Health Stat 1998;13:1-99.

7. Stange KC, Zyzanski SJ, Jaen CR, et al. Illuminating the "black box": a description of 4454 patient visits to 138 family physicians. J Fam Pract 1998;46:377-89.

8. Depression Guideline Panel. Depression in Primary Care: Vol. 1. Detection and Diagnosis. Clinical Practice Guideline, No. 5. 1993, US Department of Health and Human Services: Rockville, MD.

9. Simon GE, VonKorff M. Recognition, management, and outcomes of depression in primary care. Arch Fam Med 1995;4:99-105.

10. Judd LL. The clinical course of unipolar major depressive disorders. Arch Gen Psychiatry 1997;54: 989-91.

11. Glass RM. Treating depression as a recurrent or chronic disease. Jama 1999;281:83-4.

12. Ludman E, Von Korff M, Katon W, et al. The design, implementation, and acceptance of a primary care-based intervention to prevent depression relapse. Int J Psychiatry Med 2000;30:229-45. 
13. Lin P, Campbell DG, Chaney EF, et al. The influence of patient preference on depression treatment in primary care. Ann Behav Med 2005;30:164-73.

14. Dwight-Johnson M, Unutzer J, Sherbourne C, Tang LQ, Wells KB. Can quality improvement programs for depression in primary care address patient preferences for treatment? Med Care 2001;39:934-44.

15. Von Korff M, Goldberg D. Improving outcomes in depression. BMJ 2001;323:948-9.

16. U.S. Preventive Services Task Force. Screening for depression: recommendations and rationale. Ann Intern Med 2002;136:760-4.

17. Katon W, Von Korff M, Lin E, et al. Collaborative management to achieve treatment guidelines: impact on depression in primary care. JAMA 1995;273:1026-31.

18. Katzelnick DJ, Simon GE, Pearson SD, et al. Randomized trial of a depression management program in high utilizers of medical care. Arch Fam Med 2000;9:345-51.

19. Simon GE, Katon WJ, VonKorff M, et al. Costeffectiveness of a collaborative care program for primary care patients with persistent depression. Am J Psychiatry 2001;158:1638-44.

20. Simon GE, Manning WG, Katzelnick DJ, et al. Cost-effectiveness of systematic depression treatment for high utilizers of general medical care. Arch Gen Psychiatry 2001;58:181-7.

21. Jorm AF, Griffiths KM, Christensen H, et al. Providing information about the effectiveness of treatment options to depressed people in the community: a randomized controlled trial of effects on mental health literacy, help-seeking and symptoms. Psychol Med 2003;33:1071-9.

22. Griffiths KM, Christensen H, Jorm AF, Evans K,
Groves C. Effect of web-based depression literacy and cognitive-behavioural therapy interventions on stigmatising attitudes to depression: randomised controlled trial. Brit J Psychiatry 2004;185:342-9.

23. Grime J, Pollock K. Information versus experience: a comparison of an information leaflet on antidepressants with lay experience of treatment. Patient Educ Couns 2004;54:361-8.

24. Sandelowski M. Whatever happened to qualitative description?. Res Nurs Health 2000;23:334-40.

25. Collins KA, Westra HA, Dozois DJA, Burns DD. Gaps in accessing treatment for anxiety and depression: challenges for the delivery of care. Clin Psychol Rev 2004;24:583-616.

26. Unutzer J. Diagnosis and treatment of older adults with depression in primary care. Biol Psychiatry 2002;52:285-92.

27. Schwenk TL. Diagnosis of late life depression: the view from primary care. Biol Psychiatry 2002;52: 157-63.

28. Docherty JP. Barriers to the diagnosis of depression in primary care. J Clin Psychiatry 1997;58 Suppl 1:5-10.

29. Brody DS, Khaliq AA, Thompson TL, II. Patients' perspectives on the management of emotional distress in primary care settings. J Gen Intern Med 1997;12:403-6.

30. Dwight-Johnson M, Sherbourne CD, Liao D, Wells KB. Treatment preferences among depressed primary care patients. J Gen Intern Med 2000;15:52734.

31. Cooper LA, Gonzales JJ, Gallo JJ, et al. The acceptability of treatment for depression among AfricanAmerican, Hispanic, and white primary care patients. Med Care 2003;41:479-89. 